Mens

revue d'histoire intellectuelle de l'Amérique française

\title{
Une figure du catholicisme social canadien-français de l'entre-deux-guerres : le père Joseph-Papin Archambault, s.j.
}

\section{Frédéric Boily}

Volume 1, numéro 2, printemps 2001

URI : https://id.erudit.org/iderudit/1024447ar

DOI : https://doi.org/10.7202/1024447ar

Aller au sommaire du numéro

Éditeur(s)

Centre de recherche en civilisation canadienne-française

ISSN

1492-8647 (imprimé)

1927-9299 (numérique)

Découvrir la revue

Citer cet article

Boily, F. (2001). Une figure du catholicisme social canadien-français de l'entre-deux-guerres : le père Joseph-Papin Archambault, s.j. Mens, 1(2), 141-161. https://doi.org/10.7202/1024447ar
Résumé de l'article

Le père Joseph-Papin Archambault, s.j. (1880-1966) est avant tout un homme d'action qui s'implique dans de nombreux mouvements comme l'École sociale populaire, dont il est le directeur de 1929 à 1959. Figure centrale du catholicisme social, le père Archambault, un Jésuite, a une conception " offensive » du catholicisme. S'inspirant des encycliques papales, il mène une lutte acharnée contre la doctrine communiste, mais aussi contre le libéralisme et l'individualisme. Ces derniers sont accusés d'avoir plongé la société des années trente dans une grave crise qui serait morale avant d'être économique. Pour la surmonter, Archambault propose une réforme spirituelle, laquelle devrait conduire à celle des institutions ainsi qu'à l'établissement du corporatisme. Enfin, il croit à l'indissolubilité du lien entre le catholicisme et le nationalisme. 


\title{
UNE FIGURE DU CATHOLICISME SOCIAL CANA- DIEN-FRANÇAIS DE L'ENTRE-DEUX-GUERRES : LE PÈRE JOSEPH-PAPINARCHAMBAULT, s.j.
}

\author{
Frédéric Boily \\ Département des sciences humaines \\ Université du Québec à Chicoutimi
}

\section{RÉSUMÉ}

Le père Joseph-Papin Archambault, s.j. (1880-1966) est avant tout un homme d'action qui s'implique dans de nombreux mouvements comme l'École sociale populaire, dont il est le directeur de 1929 à 1959 . Figure centrale du catholicisme social, le père Archambault, un Jésuite, a une conception «offensive» du catholicisme. S'inspirant des encycliques papales, il mène une lutte acharnée contre la doctrine communiste, mais aussi contre le libéralisme et l'individualisme. Ces derniers sont accusés d'avoir plongé la société des années trente dans une grave crise qui serait morale avant d'être économique. Pour la surmonter, Archambault propose une réforme spirituelle, laquelle devrait conduire à celle des institutions ainsi qu'à l'établissement du corporatisme. Enfin, il croit à l'indissolubilité du lien entre le catholicisme et le nationalisme.

\section{ABSTRACT}

Priest, sociologist and social activist, Father Joseph-Papin Archambault, s.j. (1880-1966) was active in various French Canadian social movements, including the École sociale populaire, of which be was the director from 1929 to 1959. As a central figure of Canadian social Catholicism, Father Archambault, a Jesuit, had an "offensive" (as opposed to defensive) conception of Catholicism. Inspired by papal encyclicals, be fought the "pernicious" doctrines of communism, liberalism and individualism. In the thirties, be felt that the latter two had plunged society into a deep crisis that was primariby moral rather than economic. To counter this crisis, Archambault put bis faith in spiritual reforms and in corporatism. Like many French Canadian intellectuals of his era, he firmly believed in the indissoluble link between nationalism and Catholicism. 
Le père Georges-Henri Lévesque, décédé il y a peu et fin connaisseur du milieu intellectuel canadien-français dont il fut l'un des acteurs les plus importants, affirme dans ses Souvenances que le directeur de l'École sociale populaire (ÉSP) pendant les années trente, le Jésuite Joseph-Papin Archambault, fut un grand catholique social. «Dans ma vie, se remémore le père Lévesque, j'ai connu deux mordus de la doctrine sociale, deux personnes qui ont fini par s'identifier à elle en quelque sorte : le père Archambault et monsieur Desrosiers [un Sulpicien] ${ }^{2} »$. Un survol même rapide des textes du premier amène à croire à la justesse de son appréciation. En effet, les références aux encycliques papales, principalement Rerum novarum (1891) et Quadragesimo anno (1931), sont nombreuses au point de constituer l'armature principale des textes du directeur de l'ÉSP. Mais plus précisément, qu'est-ce que cela veut dire d'être un catholique social dans le Québec des années trente ${ }^{3}$ ? Surtout lorsque l'on garde à l'esprit que la doctrine sociale de l'Église ne constituait pas un système arrêté d'idées. Comme l'explique l'historien Jean-Marie Mayeur, si l'encyclique Rerum novarum, pierre de touche du catholicisme social, est «ferme sur les principes et les orientations», elle reste ouverte sur les applications et les solutions ${ }^{4}$, donc ouverte à la contextualisation. Dans ces conditions, quelle est la nature du catholicisme d'Archambault? Certes, ce dernier apparaît plutôt être un homme d'action qu'un intellectuel à la Lionel Groulx. Mais son engagement dans la cité devait certainement reposer sur un certain sol théorique. Cette étude vise donc à offrir un portrait intellectuel d'un acteur important, quoique moins connu, du clérico-nationalisme des années trente.

Avant de s'intéresser plus particulièrement à la pensée d'Archambault, un détour est nécessaire pour exposer les grandes lignes du projet catholique dans la première moitié du $\mathrm{XX}^{\mathrm{e}}$ siècle. Plus exactement, il s'agira de brosser un rapide tableau du catholicisme social européen, celui-ci ayant servi de matrice intellectuelle au catholicisme québécois ${ }^{5}$. On verra que le père Archambault avait une conception «offensive» du catholicisme, c'est-à-dire que le prêtre, loin d'être cantonné à la sacristie, devait au contraire se jeter dans la mêlée pour lutter contre le communisme et le libéralisme de manière à se rapprocher du peuple. On constatera que, sur le fond, sa doctrine reste dans l'orbite du catholicisme le plus critique de la modernité, celui-là même qui cherche à imposer dogmatiquement une régulation religieuse de la société dans le courant du $\mathrm{XIX}^{e}$ siècle. 


\section{Définition du catholicisme social}

Le premier élément à mettre en relief à propos du catholicisme social, c'est son caractère antilibéral qu'il tient de ses origines liées

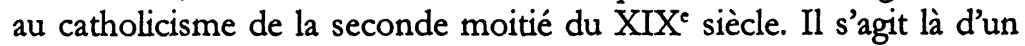
point important, car il faut bien se rendre compte que la matrice intellectuelle du catholicisme social est celle du catholicisme le plus dur envers la modernité, le «catholicisme intransigeanb», pour reprendre la terminologie d'Émile Poulat dans son ouvrage Église contre bourgeoisie'. En effet, comme l'explique Poulat, l'encyclique Rerum novarum, pierre de touche du catholicisme social, n'est pas venue supprimer le Syllabus', mais plutôt l'approfondir ${ }^{8}$. Or, «l'intransigeantisme» catholique oppose une attitude radicale de refus du monde moderne, et implique l'idée qu'il faut impérativement «recatholicisen» la société. L'historien JeanMarie Mayeur souligne bien également l'ambiguité constitutive du catholicisme social français, lorsqu'il écrit que si les catholiques sociaux acceptent la démocratie et la République, ils n'acceptent pas le libéralisme et ils partagent «la vision du monde qui était celle des intransigeants $\left.{ }^{2}\right)$. En réalité, la distinction entre catholicisme intransigeant et catholicisme social n'est pas une différence de nature, mais plutôt d'attitude à adopter face à la nouvelle société. Il vaut donc mieux parler, comme Poulat le fait, d'un «nouvel intransigeantisme» : «Le vieil intransigeantisme était une attitude de vaincus, qui sauvaient l'honneur après avoir perdu la guerre, écrit Poulat. Le nouvel intransigeantisme entend reprendre l'initiative; il a l'âme conquérante, et à un catholicisme de position, il veut substituer un catholicisme de mouvement ${ }^{\circ}$ \%. D'un catholicisme arc-bouté sur la défense têtue du dogme, on passe à un catholicisme qui a toujours une vision intransigeante du monde, mais dont l'instinct stratégique, plus développé, l'amène à vouloir reconquérir les masses.

La vision du monde véhiculée par le catholicisme social était cependant toujours organisée autour de la philosophie organiciste, $\mathrm{du}$ refus de l'individualisme ainsi que du rêve d'une alliance entre le peuple et les élites cléricales, le tout se pensant comme une "troisième voie» entre le libéralisme et le communisme" ${ }^{1}$. En d'autres termes, on retrouvait chez les catholiques sociaux ce qui constituait le fonds de commerce de l'intransigeantisme, c'est-à-dire le urefus des compromissions et des accommodements avec le siècle des catholiques libéraux [...]", l'ultramontanisme et surtout, ce qui s'avère fondamental pour comprendre Archambault, la «volonté d'une action catholique dans le monde [ainsi que le] refus d'une conception qui séparait le religieux du profane et interdisait au prêtre de sortir de la sacristie 
$[\ldots]^{12} \gg$. Au fondement de ces deux courants du catholicisme, on retrouve la même préoccupation de contrôler religieusement les masses :

On est donc en présence de deux modèles adverses se référant au même système avec la même intention de la maintenir, l'un en l'adoptant à des conditions nouvelles, l'autre en le restaurant dans son ancienne situation [...]. De leur commune origine, ils ont gardé le même projet fondamental : une régulation religieuse de la société globale ${ }^{13}$.

Ainsi, le catholicisme social ne constituerait nullement un affadissement de la doctrine catholique, car il est aussi à sa manière une tentative de reconquête et de recatholicisation de la société, mais à partir d'une nouvelle stratégie de rapprochement avec le peuple. Cependant, les catholiques sociaux sont aux prises avec de nouveaux adversaires, le socialisme et le communisme, lesquels leur disputent leur influence sur le peuple. «Le libéralisme, écrit Hugues Portelli, avait pour conséquence immédiate de menacer le lien traditionnel entre Église et classes dirigeantes, entre pouvoir religieux et pouvoir civil. Le socialisme s'attaque à son influence séculaire sur le peuple ${ }^{14} »$. En effet, si le catholicisme de 1880 luttait contre le seul libéralisme, le catholicisme social du début du $\mathrm{XX}^{\mathrm{e}}$ siècle est également confronté au socialisme et au communisme. On assiste donc à une lutte triangulaire où l'Église doit lutter à la fois contre le libéralisme et le socialisme/communisme pour instaurer son projet de régénération chrétienne de la société. Si l'ennemi numéro un est bien, dans les années trente, le communisme, il ne faut pas oublier l'importance de la lutte contre le libéralisme. Car l'Église affirmait, comme l'explique l'historien et soviétologue Alain Besançon, que «la responsabilité première dans la genèse du communisme» devait être imputée au libéralisme ${ }^{15}$. Ainsi que le rappelle JeanMarie Mayeur, l'Église ne s'allie pas à la bourgeoisie, car elle ne cesse pas de dénoncer l'hypocrisie sur laquelle, selon elle, le monde bourgeois repose : «Loin de s'adapter au monde moderne, l'Église de Pie IX à Paul VI, tient un même discours, lorsqu'elle dénonce «une civilisation mercantile, hédoniste, matérialiste», selon les mots de Paul VI pour la Pentecôte de $1975^{16}$ ». Bref il existerait, entre l'Église, le libéralisme et le socialisme un "conflit triangulaire», pour reprendre l'expression d'Émile Poulat, mettant aux prises trois armées : «la rouge, la blanche et la noire ${ }^{17} »$. Voilà les grandes lignes de ce qui nous semble être les principaux éléments théoriques du catholicisme social tel qu'il s'est exprimé dans le contexte européen, mais nécessaires pour comprendre Archambault. On verra entre autres choses qu'en bon catholique social, le directeur de l'ÉSP lutte à la fois contre le libéralisme et le communisme. 


\section{Lutte contre le communisme et le libéralisme}

L'ÉSP a surtout laissé sa marque dans la lutte anticommuniste. Comme l'ont bien noté quelques auteurs (André J. Bélanger et Gilles Routhier, par exemple), les textes anticommunistes occupent une large place dans le corpus doctrinal de l'ÉSP. À cet égard, le père Joseph-Papin Archambault a été tout particulièrement actif. Il deviendra même, comme le rappelle l'historienne Lucia Ferretti, informateur de la police provinciale et de la Gendarmerie royale ${ }^{18}$. S'il craint tant la menace rouge, c'est qu'elle entraîne à sa suite «ruines matérielles et morales ${ }^{19}$ ). Théologiquement, il considère le communisme injuste, parce qu'il viole le droit naturel de propriété, et néfaste, parce qu'il "détruit la famille [et] éteint dans l'âme tout sentiment religieux ${ }^{20}$ 》. Il est donc du devoir de tout bon catholique de combattre une doctrine aussi perverse. Dans une brochure de l'ÉSP consacrée au compte rendu des premières Journées anticommunistes, Archambault affirme qu'il faut lutter contre la "vague de feu» déferlant sur le monde ${ }^{21}$. Car bien que jusqu'ici, grâce à sa position géographique, le Canada ait été épargné, la menace rouge pointe à l'horizon, notamment chez les populations immigrées. Archambault ne pense pas seulement aux immigrants des «immenses plaines de l'Ouest), pour reprendre son expression, mais également, et surtout, à ceux de l'Abitibi et de Montréal ${ }^{22}$. À cet égard, la situation de Montréal l'inquiète tout particulièrement parce que, explique-t-il, dans une CEuvre des tracts, la ville «se cosmopolise [...] rapidement» tout en accueillant une population des «classes pauvres ${ }^{23}$ ). Il faut bien se rendre compte que la critique du communisme se double d'une critique de l'immigration, puisque les immigrants sont accusés d'être majoritairement favorables à «l'idéologie rouge». Montréal «renferme un grand nombre d'immigrants de nationalités diverses, écrit Archambault, dont de forts groupes de l'Europe centrale, déjà gagnés pour la plupart au bolchevisme avant d'entrer au pays ${ }^{24}$ ). La lutte contre le communisme devrait donc passer par un contrôle plus serré des frontières.

C'est pourquoi, selon Archambault, l'intervention de l'État est une nécessité de première importance pour lutter contre le communisme, ce qui pourrait surprendre, puisque l'on affirme généralement que l'ÉSP est apolitique ${ }^{25}$. Selon Archambault, l'État a le devoir de défendre la société contre l'anarchie provoquée par le communisme au sein de la société. L'État doit exercer avec une grande fermeté ses fonctions de police, le communisme étant une grave menace pour le bien commun. En conséquence, les autorités fédérale, provinciales et municipales ne doivent pas hésiter à utiliser l'arme judiciaire. 
C'est aussi, croit Archambault, parce qu'il est «armé» que l'État a l'obligation morale de se défendre contre ceux qui menacent de le renverser : «Encore que ce ne soit pas le moyen idéal, explique-t-il, la manière forte est nécessaire dans certaines circonstances ${ }^{26}$ ). Affirmant que «[]'action de l'État [...] paraît la première arme à employer ${ }^{27} »$, Archambault loue l'Article 98 du Code criminel dont il souhaite «'application rigoureuse» à travers le pays. Ainsi, il ne souhaite rien de moins que restreindre, sinon enlever, la liberté d'expression et de parole aux communistes. «Atteinte à la liberté dira-t-on? Mais depuis quand le mal, le désordre, l'anarchie ont-ils des droits ${ }^{28}$ ?» $\grave{A}$ ses yeux, le communisme est donc un «poison» dont l'existence ne peut être tolérée. L'État devrait également compléter son arsenal juridique avec des dispositions contre les immigrants, car, comme on l'a vu plus haut, ceux-ci sont vus par le directeur de l'ÉSP comme les principaux propagateurs des thèses communistes. "C'est parce que nous avons ouvert nos portes toutes grandes aux immigrants que le communisme s'est installé chez nous. Il faut maintenant les tenir fermées, puis refuser la naturalisation à tout étranger suspect de tendances communistes, expulser même les agitateurs ${ }^{29}$ ). La société canadiennefrançaise doit se barricader derrière ses frontières, sinon elle risque de voir déferler une immigration que l'on croyait favorable au communisme ${ }^{30}$. L'État devait donc, selon Archambault, se faire gendarme et assurer un fort contrôle aux frontières.

En raison de la place importante occupée par la dénonciation du communisme, on pourrait croire que celui-ci constitue le seul et unique ennemi de l'ÉSP. Or, sans minimiser aucunement l'importance du thème de la menace communiste, on ne s'est pas assez interrogé sur la généalogie de la crise telle que la percevait Archambault. Celui-ci semble en effet avoir repris la vision véhiculée par l'Église, exposée plus haut, voulant que le communisme soit une créature infernale sortie du ventre de la bête libérale. Comme on va le voir, il ne cesse de condamner l'individualisme, et il affirme à de nombreuses reprises que les raisons du succès communiste sont à chercher du côté des méfaits du libéralisme, plus précisément de l'individualisme, présenté comme un égoïsme destructeur.

Cela me permet de corriger la thèse, avancée surtout par l'historienne Fernande Roy, voulant que la doctrine sociale de l'Église, notamment celle de l'ÉSP, était un cryptolibéralisme en raison de la défense qu'elle fait de la propriété privée. Dans Progrès, harmonie, liberté, elle affirme entre autres choses que si la doctrine sociale de l'Église se présentait comme une recherche de "troisième voie» entre 
le libéralisme et le socialisme, elle constituait, au fond, davantage une humanisation du libéralisme qu'un rejet des principes libéraux. $\mathrm{Si}$ bien que l'idéologie libérale n'aurait pas été véritablement ébranlée ${ }^{31}$. Selon Roy, «l'Église respecte absolument la propriété privée», encore que l'historienne reconnaisse que l'Église n'y attache pas le même sens que les libéraux. Sans remettre en cause l'existence d'un courant libéral au sein de la société québécoise, je ne suis cependant pas du tout convaincu que la pensée de l'Église ait été séduite par le libéralisme ${ }^{32}$ et que, somme toute, comme le pense Roy, la fameuse «troisième voie» ne s'éloignerait pas beaucoup des libéraux ${ }^{33}$.

L'élément m'apparaissant décisif pour rejeter la thèse de Roy concerne le critère retenu par celle-ci pour qualifier le libéralisme. A mon avis, le respect de la propriété privée ne constitue pas un bon critère pour identifier le libéralisme. Et cela parce que l'on peut respecter la propriété privée et vouloir récolter les fruits de la prospérité économique tout en étant antilibéral, c'est-à-dire rejeter la philosophie des Lumières et les principes, comme l'autonomie individuelle, sur lesquels repose le libéralisme politique. Le meilleur exemple que l'on puisse donner de cette attitude est l'idéologie fasciste, laquelle, tout en étant profondément contre le libéralisme politique, ne voulait pas abolir la propriété privée. Ainsi que l'écrit l'historien Zeev Sternhell, la urévolution fasciste entend changer la nature des rapports entre l'individu et la collectivité sans pour autant briser le moteur de l'activité économique - la recherche du profit -, ni abolir son fondement - ou détruire son cadre nécessaire - l'économie de marché ${ }^{34} »$. L'exemple fasciste montre que l'antilibéralisme peut s'exprimer tout en respectant les principaux postulats du capitalisme et du libéralisme économique. Ou, si on préfère, que l'on peut être plus ou moins libéral en matière économique et fortement opposé au libéralisme politique. En somme, on ne peut se contenter d'affirmer qu'une pensée est libérale parce qu'elle reconnaît certains principes du capitalisme et du libéralisme économique.

Pour revenir à Archambault, on constate, en conformité avec les grandes orientations idéologiques de l'Église, qu'il accuse continuellement le libéralisme d'être à l'origine de la crise et du communisme. Ironiquement, l'explication proposée est similaire à celle du communisme. En effet, quand il explique la genèse de la crise durant la Semaine sociale consacrée à l'organisation professionnelle (en 1936), le directeur de l'ÉSP, faisant largement appel à des extraits de Quadragesimo anno, explique que Pie XI a constaté «l'accumulation d'une énorme puissance aux mains d'un petit nombre d'hommes» ${ }^{35}$. 
Ensuite, que cette concentration au sein d'un petit nombre d'hommes «est le fruit naturel d'une concurrence dont la liberté ne connaît pas de limites $\left.[. . .]^{36}\right)$. Enfin, que cette concurrence effrénée pour l'accumulation des ressources aurait conduit finalement à la lutte pour la domination mondiale, ladite lutte empruntant le chemin suivant : (i) combat pour la maîtrise économique; (ii) le combat se déplace sur le terrain du politique; (iii) la lutte finit par opposer des États qui se battent pour des intérêts économiques ${ }^{37}$. Cette interprétation, comme le fait remarquer Alain Besançon, est précisément celle du communisme - l'Impérialisme stade suprême du capitalisme - laquelle imputait une cause économique aux luttes sanglantes que se livraient les États ${ }^{38}$. Paradoxalement, Archambault, s'inspirant de Pie XI, pensait à partir du cadre mental mis en place par le socialisme. En d'autres termes, le libéralisme, à l'en croire, aurait mis en place les conditions sociales ayant favorisé l'expansion de la «menace rougen, pour parler à la manière du directeur de l'ÉSP.

À cela Archambault ajoute une virulente critique de l'individualisme. En effet, il écrit en 1932 dans un petit opuscule qu'il faudra prémunir la jeunesse contre «l'égoïsme [...] à la base de tous nos maux ${ }^{39}$ ». Dans le même opuscule, Archambault explique que Léon XIII a condamné, avec Rerum novarum, autant le libéralisme que le socialisme puisqu'il y a une "condamnation» de «la doctrine du laisser faire ${ }^{40}$ ». Cette condamnation du «laisser faire» se justifie, selon le directeur de l'ÉSP, dans la mesure où la doctrine sociale de l'Église exige «que toute l'économie de la vie domestique soit réglée d'après les commandements de Dieu et les principes de la loi naturelle $[. . .]^{41} \gg$. En ce sens, l'Église, explique Archambault, ne pouvait entériner les principes du «laisser faire» parce que cela revenait pour elle à accepter la thèse selon laquelle elle n'aurait rien eu à dire dans l'organisation de la société. En d'autres termes, l'économie ne constitue pas une sphère autonome de la morale chrétienne. En 1934, dans la brochure déjà citée consacrée à la lutte anticommuniste, Archambault rappelle que «le Pape condamne plus encore l'individualisme que le communisme parce que celui-ci est né de celui-là ${ }^{42}$ ). Ce serait donc dans l'individualisme que s'enracineraient les causes profondes de la naissance du communisme.

Archambault développe notamment cette idée durant la Semaine sociale de 1935, lorsqu'il explique que la crise ébranlant les sociétés occidentales, et plus précisément celle touchant les classes ouvrières, est avant tout celle du libéralisme individualiste. Comme il le dit, le mal «dont souffre profondément notre siècle [c'est] l'individualisme ${ }^{43}$ ». Ce demier, selon lui, exerce «ses ravages dans les domai- 
nes économique et politique, dominant l'industrie, la spéculation commerciale et les manipulations bancaires $[\ldots]^{44}$ ", tout en empêchant également les classes ouvrières de s'organiser. Et cela est aussi vrai, affirme Archambault, pour les électeurs et les gouvernants. $\mathrm{Si}$ le libéralisme est si pernicieux, c'est qu'il empêche les forces vives de la société de s'organiser. Comme l'explique le directeur de l'ÉSP dans sa déclaration d'ouverture à la Semaine sociale de 1936, le libéralisme aurait détruit la vie sociale (il cite ici Pie XI), «qui s'épanouissait jadis en une riche et harmonieuse floraison de groupements les plus divers ${ }^{45}$ ). En ce sens, l'individualisme est stigmatisé parce qu'il est venu mettre fin à la vie organique animant la société. En somme, le libéralisme atomise la société et la «libre concurrence», son principe directeur, fait que «l'appétit du gain» domine tout ${ }^{46}$. Voilà le terreau créé par le libéralisme.

Or, selon Archambault, c'est précisément dans ce terreau, comme il l'explique dans le journal L'Ordre nouveau, que le communisme s'épanouit : «L'individualisme fait le lit du communisme. [...] La force du communisme réside dans les abus du capitalisme[... ${ }^{47}$ »). En ce sens, le communisme constitue l'enfant bâtard de l'individualisme. C'est pourquoi une des tâches importantes des Semaines sociales consiste à lutter contre «'individualisme congénital», expression empruntée à Olivar Asselin, qui "paralyse notre activité et fait de nous dans un riche domaine des propriétaires pauvres ${ }^{48}$ ). Mais que faire devant les désastres occasionnés par l'individualisme, perçu comme néfaste, et le communisme?

\section{La crise spirituelle : de la réforme des esprits à celle des institutions}

Dans l'optique où l'individualisme est sans cesse accusé d'être à l'origine de la crise, celle-ci acquiert une nature particulière, c'est-àdire que la crise, avant d'être sociale et politique, est avant tout spirituelle. En effet, en 1932, lors de la Semaine sociale qui mettait à l'honneur le thème de L'ordre social chrétien, Archambault affirme que si le régime capitaliste a conduit à l'inégale répartition des richesses, son plus grand tort est surtout d'avoir dégradé la spiritualité du peuple : «Ruines matérielles incalculables! Mais combien plus profondes et plus navrantes encore les ruines spirituelles $!^{49}$ " Il faut bien se rendre compte que le sentiment d'une grave menace pesant sur la civilisation chrétienne était très poussé chez Archambault. Comme il le dit, cette fois à la Semaine sociale de 1938, si la menace est lourde de conséquences, c'est parce qu'il en va de la survie même des âmes : 
«Un péril mortel menace la société, la civilisation chrétienne est en danger, les âmes vont périr ${ }^{50} \%$. Certes, une telle affirmation rend un drôle de son aujourd'hui, mais elle indique clairement que la crise est spirituelle avant d'être matérielle et institutionnelle. Cette nature particulière pousse d'ailleurs Archambault à préconiser certains types de solutions, plutôt que d'autres.

Il affirme ainsi que «sans une complète rénovation de l'esprit chrétien», c'est la course à l'abîme ${ }^{51}$. En effet, il était persuadé que «[s]eule une réforme des mœurs, une rationalisation morale préviendra les catastrophes menaçantes et restaurera l'ordre social désemparé. Car c'est d'elle uniquement que sortiront les réformes économiques nécessaires $^{52}$ \%. Avant d'entreprendre des réformes de nature institutionnelle, il faut d'abord procéder à une rénovation spirituelle et s'attaquer à «l'égoïsme sans frein» qui mine la société ${ }^{53}$. Archambault croyait fermement que la crise ne saurait être surmontée sans faire appel aux enseignements spirituels contenus dans l'Évangile : «Une fois de plus, sur le monde en détresse s'affirme, comme seul et suprême espoir, la primauté des forces spirituelles ${ }^{54} \%$. Plus exactement, cette réforme des mœurs implique une «substitution de la modération chrétienne à l'égoïsme sans frein qui est la honte et le grand péché de ce siècle $[. . .]^{55}$ \%. Archambault voit donc la crise comme une question morale», «une défaillance des âmes» avant de la voir comme une défaillance structurelle du capitalisme ${ }^{56}$, même si cet aspect est aussi présent chez lui, comme on l'a vu plus haut.

C'est pourquoi il faut d'abord avant tout uredressen» et «spiritualisen» les âmes, afin de créer une génération «d'élites vraiment chrétiennes", pour reprendre les mots du cardinal Pacelli (futur Pie XII) cité par Archambault, en 1932 : «Non pas nécessairement, du premier coup, toutes, mais quelques-unes, choisies, groupées et qui, placées aux points critiques de l'organisme social, lui infuseront cet esprit vital sans lequel il s'affaisse et tombe en ruine. ${ }^{57}$ " Cela dénote une conception élitiste de la vie sociale, car il est supposé implicitement que les masses doivent être menées par des élites capables de les guider en direction de verts pâturages. En 1935, Archambault explique donc que les Semaines sociales, pour briser «les lourdes chaînes de l'individualisme ${ }^{58}$ ), ont pour mission fondamentale de favoriser la création «des hommes d'élite» nécessaires à l'émergence du monde nouveau espéré.

Mais Archambault veut aussi s'occuper des masses, car «[p]lus encore que de pain, les foules aujourd'hui ont besoin de doctrine ${ }^{59} \%$. 
Pour ce faire, il insiste sur l'éducation. À cet égard, Archambault est tout à fait dans l'air du temps. "Qu'il s'agisse des menaces extérieures ou des ressources du dedans, expliquait Fernand Dumont à propos des idéologies des années trente, le problème essentiel et la solution suprême relèvent de la formation des hommes, de l'éducation $^{60} \%$. L'abbé Groulx, par exemple, a consacré de nombreuses pages à la nécessité d'instaurer un type d'éducation nationale propre à ranimer le sens national, qu'il jugeait anémié, de ses compatriotes ${ }^{61}$. Archambault croit lui aussi à l'importance de l'éducation, car le «désordre grandissant» qui affecte la société est un «désordre dans les mœurs, les institutions, les lois». En ce sens, seule une «réforme des esprits» sera, estime-t-il, en mesure de contrer le désordre dans la société. Et ce parce que «le désordre social provient du désordre intellectuel comme l'effet à la cause ${ }^{62} \geqslant$. D'où la nécessité d'une réforme de l'éducation. Mais contrairement à Groulx qui parlait d'éducation nationale, Archambault insiste plutôt sur la notion "d'éducation sociale», "œuvre» essentielle à ses yeux et «dont dépend l'avenir même de la civilisation chrétienne ${ }^{63}$ ). Comment définit-il cette éducation sociale?

Au premier chef, il s'agit d'une éducation combattant l'ennemi que constitue l'individualisme. L'éducation doit en effet avoir pour objectif, comme l'écrit Archambault dans L'Ordre nouveau (en parlant de l'éducation que les mères doivent prodiguer à leurs enfants), de faire sortir l'individu «de son affreux petit moi ${ }^{64}$ ». C'est pourquoi «[]]'éducation sociale, affirme-t-il, fera agir socialement. Elle enseignera les devoirs envers le prochain, elle entraînera les âmes à la pratique des collaborations nécessaires, elle assignera comme but principal à leurs énergies non la poursuite de l'intérêt personnel mais le service du bien commun ${ }^{65}$ ). Une telle éducation devra, pense-t-il, être prodiguée tout au long de la vie de l'individu, c'est-à-dire à partir de l'enseignement maternel jusqu'au terme de l'existence en passant par la «petite école» et l'université. C'est ainsi que les Semaines sociales font également partie de l'effort d'éducation qu'il convient d'entreprendre. Ceci est également vrai pour la profession et l'Église qui doivent devenir des lieux d'éducation sociale tout comme l'Action catholique qui, aux dires d'Archambault, constitue «un puissant agent d'éducation ${ }^{66} \%$. Au total, toutefois, il n'en dit guère plus sur le contenu d'une telle éducation.

On précisera qu'il ne s'agit pas ici d'une éducation voulant favoriser l'autonomie intellectuelle, dans une optique inspirée de Kant par exemple, mais plutôt d'une éducation qui veut donner des principes fermes agissant comme des bouées de sauvetage dans le 
tumulte de la vie intellectuelle. C'est ce que l'on constate lorsque Archambault explique ce qu'il espère des conférenciers venus livrer leur savoir aux Semaines sociales. Ces propos sont révélateurs, me semble-t-il, de la philosophie sous-tendant sa conception de l'éducation. Les conférenciers invités aux Semaines sociales, explique-t-il, "parlent en maitres [et] leurs cours ne souffrent pas de discussion publique ${ }^{67} 1$. L'éducation n'est pas ici vue comme un instrument favorisant le développement de l'autonomie individuelle, mais comme l'inculcation d'une manière de penser, puisque ceux qui viennent aux Semaines sociales «s'en retourne[nt] avec une doctrine bien claire, bien nette, bien homogène ${ }^{68} \%$. Au total, l'éducation est comprise par Archambault comme une entreprise de combat contre «les excès de l'individualisme».

En fait, il ne s'agit pas tant d'éduquer que de faire prendre conscience aux individus qu'ils doivent apprendre à respecter leur place sur l'échiquier social. Archambault appelle cela une "propagande constructive». Par exemple, explique-t-il, en 1935, "pour éclairer l'ouvrier et l'attacher à ses traditions, [il importe] de lui faire connaitre la doctrine sociale catholique : l'inégalité des conditions, la nécessité de la souffrance, le rôle véritable de la richesse $[. . .]^{69}$ ). Au diapason de l'idéologie organiciste - laquelle présume que les sociétés sont des totalités sociales ayant leur vie propre - il s'agit d'inculquer à l'individu l'idée qu'il n'est qu'une cellule d'un grand corps. Ainsi, la subordination individuelle à la totalité sociale est naturelle et voulue par Dieu. Bref, à la doctrine communiste qui propose un avenir radieux exempt de la domination de classe, l'ÉSP et Archambault opposent la doctrine de la soumission à la totalité sociale.

Archambault estime également que l'apostolat laïc, dont l'Action catholique est une des composantes, est essentiel dans l'effort de rénovation spirituelle et morale de la société. D'abord, pour lutter contre le communisme et pour maîtriser le supposé individualisme excessif des Canadiens français, «l'apostolat intellectuel» est sollicité au premier chef. Cet apostolat, qui consiste à «éclairer les intelligences», constituait, aux dires mêmes d'Archambault, l'un des objectifs avoués de l'ÉSP ${ }^{70}$. Ensuite, l'apostolat laique, rune des gloires de notre siècle ${ }^{71}$ ", signifie, selon Archambault, qu'il faut être un véritable apôtre qui ne se contente pas «d'accomplir de son mieux ses devoirs de religion, mais [qui] s'efforce [...] d'entraîner dans la même voie le plus d'âmes possible $[\ldots]^{73}$ ). Il faut bien voir la forte volonté d'action et de combat sous-tendant cet apostolat, car il ne s'agit pas seulement de convaincre par la parole. En effet, le directeur de l'ESP donne en exemple Clovis, lequel aurait affirmé que, s'il avait été 
présent lors de la crucifixion du Christ, il l'aurait «bien vengé!73, La métaphore guerrière est également présente lorsque Archambault parle de l'action catholique, plus précisément quand il explique que le curé est "chef» des paroisses, celles-ci étant les «cellules de notre corps nationaly, et que les diverses associations de l'Action catholique «sont ses troupes d'élite, son état-majon. Et ces troupes «combattent loyalement sous ses ordres, dévouées à l'Église, ardente pour la gloire de Dieu $^{74}$ ).

Il y a toutefois un problème particulier qui se pose à l'égard de l'Action catholique. Jusqu'où l'apostolat laïque et l'action catholique peuvent-ils s'avancer dans la défense de la société sans tomber dans la marmite politique? Archambault est préoccupé par cette question comme on peut le voir, en 1938, dans L'Action catholique d'après les directives pontificales. Il y écrit d'abord que l'action catholique «n'est pas d'ordre temporel mais spirituel [...] ni d'ordre politique mais religieux ${ }^{75} \%$. Or, il s'empresse d'ajouter que cette caractéristique une restreint pas son champ d'action». Au contraire, affirme-t-il, l'action catholique uembrasse tout l'homme, dans la vie privée comme dans la vie publique 79 . En ce sens, et malgré les proclamations voulant que l'Action catholique relève exclusivement de l'ordre spirituel, elle le déborde pour s'inscrire nettement dans l'ordre temporel. «L'objet de l'Action catholique, écrit Archambault, est donc en quelque sorte illimitép7». On s'aperçoit en effet qu'Archambault navigue entre l'idée d'une Action catholique devant éviter l'accusation ou la tentation d'inscrire l'action catholique dans la politique et l'idée d'une action catholique qui l'amène, par sa logique propre qui est celle de recatholiciser la société, à s'immiscer de plus en plus profondément dans l'ordre temporel. Car, écrit-il, si la politique s'approchait trop du domaine de l'Action catholique (et on voit mal comment une telle chose pourrait ne pas survenir compte tenu que l'Action catholique est illimitée puisqu'il s'agit de rechristianiser la société), alors uil faudra bien qu'elle [la politique] en subisse les conséquen$\operatorname{ces}^{78}$ ». En somme, l'exemple de l'Action catholique montre bien l'ambiguité entourant le projet d'Archambault : d'un côté, le refus de la politique partisane, et de l'autre, une volonté de modifier profondément le politique défini comme l'ensemble des valeurs et des pratiques sur lesquelles repose une société (dans le langage d'aujourd'hui, on parlerait de culture politique).

Pour surmonter la crise dans laquelle la «doctrine du laisser faire» a plongé la société occidentale et pour contrer l'utopie étatiste (et païenne) issue du socialisme et du communisme, Archambault propose également une réforme des institutions. En d'autres ter- 
mes, la réforme spirituelle se double d'une réforme structurelle et institutionnelle. À la lumière des encycliques Rerum novarum, Quadragesimo anno et des théories corporatistes européennes, le directeur de l'ÉSP privilégie «le régime de l'économie contrôlée, réglementée, disciplinée ${ }^{79}$ \%. Ce contrôle, explique-t-il, seule «la profession organisée» peut l'exercer. Car, selon lui, l'établissement de la profession organisée permet de mettre fin à la lutte que se livrent les classes sociales pour l'appropriation, égoïste, des ressources. «Jamais peutêtre, écrit Archambault en parlant de l'organisation corporative, n'avons-nous traité un sujet dont les répercussions peuvent être aussi décisives pour notre avenir ${ }^{80}$ \%. Très clairement, il avance l'idée que l'organisation corporative est un système venant se substituer aux "théories malfaisantes du libéralisme économique ${ }^{81}$ ». Pour contrecarrer ces "théories malfaisantes», «le régime corporatif) ne doit pas devenir un système voué à la seule défense d'une catégorie professionnelle. Au contraire, ce régime doit permettre de sauvegarder «l'intérêt commun de toute la société ${ }^{82}$ \%.

Plus exactement, Archambault s'est fait le promoteur du corporatisme, lequel constituerait une troisième voie «entre la liberté effrénée dont nous souffrons et la domination étatiste qui nous menace $[\ldots]^{83}$ ). Selon lui, et ici il s'inspire de Firmin Bacconnier (théoricien français du corporatisme, proche de L'Action frangaise de Charles Maurras), trois principes directeurs sont au fondement de la «vieille» idée corporative, soit la «production réglée», la uproduction de qualité» et enfin, le «juste prix $)^{84}$. Essentiellement, le corporatisme a pour objectif, en disciplinant l'économie, de favoriser l'atteinte du bien commun. Il faut toutefois distinguer, explique le directeur de l'ÉSP, entre un corporatisme d'association et un corporatisme d'État. Celui-ci est une "création d'autorité» de la part d'un gouvernement, et c'est ce type de corporatisme que l'on retrouverait en Italie, en Espagne, au Portugal, en Allemagne, en Autriche et en Bulgarie ${ }^{85}$. Ce type de corporatisme est à éviter parce qu'il ne laisserait pas, selon Archambault, assez de liberté aux corps professionnels, les gouvernements imposant «leurs vues et leurs décisions ${ }^{89}$. Par conséquent, l'esprit corporatiste, lequel vise à donner plus d'autonomie aux corps professionnels, est absent. Archambault préconise donc plutôt le corporatisme d'association, car «[c]e sont les intéressés eux-mêmes, les hommes d'une même profession, d'un même milieu, qui, entraînés par les besoins des temps, par la nature de leurs intérêts, par le souci du bien commun, s'entendent pour se constituer en corporation ${ }^{87}$ ». Tel que décrit par Archambault, ce type de corporatisme semble correspondre à l'idéal ou l'utopie d'une société prenant conscience d'elle-même et de son existence et qui s'orga- 
nise pour en assurer la pérennité. On notera que la coopération remplit essentiellement la même fonction en ce qui concerne le libéralisme économique, celle-ci étant perçue par le directeur de l'ÉSP comme un élément du régime corporatif, un rouage dans une machine ${ }^{88}$ I). Tout comme le corporatisme, la coopération vise à lutter contre «les pratiques abusives du libéralisme économique $[. . .]^{89}$ ). En somme, la solution corporative et la coopération permettent de remettre sur le devant de la scène la notion de bien commun.

\section{Nationalisme et catholicisme chez Archambault}

À mon sens, un portrait intellectuel du père Archambault serait incomplet si on ne prenait pas en compte l'importance qu'il accordait au nationalisme. Selon l'historienne Lucia Ferretti, le catholicisme social d'ici avait une forte teinte nationaliste : «L'Église québécoise entreprend [...] de bâtir l'ordre social chrétien des temps urbains et industriels. Souvent avec ceux-là mêmes qui portent déjà le projet national. Ce qui confère au catholicisme social québécois sa couleur propre, nationaliste ${ }^{90}$ \%. Ainsi formulée, l'affirmation pourrait laisser croire qu'il y avait des catholiques sociaux désireux de construire un ordre social chrétien auxquels se seraient alliés des nationalistes. Or, avec Archambault, on voit plutôt que l'on ne peut tracer de frontières nettes entre catholicisme social et nationalisme. Il n'y a donc pas d'un côté des nationalistes et de l'autre des catholiques sociaux, mais des catholiques sociaux plus ou moins nationalistes.

Par exemple, dans un texte de 1928 consacré au projet de drapeau canadien-français, Archambault avance l'idée voulant que le catholicisme soit au fondement de la nation canadienne-française. En fait, ce texte nous montre que le directeur de l'ÉSP pense la nation en vertu du "principe catholicocentrique», pour reprendre la terminologie du sociologue français Yves Déloye. Selon ce dernier, on parle de "principe catholicocentrique» lorsque la nationalité et la religion forment un tout indissociable, «le lien national [étant pensé] comme consubstantiel au catholicisme ${ }^{91} \gg$. En termes simples, la nation est par essence catholique, le catholicisme en constituant, au sens fort du terme, l'âme. Pour revenir à Archambault, on voit qu'il offre une version canadienne-française de ce principe lorsque, dans son texte sur le drapeau, il croit nécessaire de mettre sur le drapeau national un symbole religieux qui, tout en venant rappeler le passé de la nation, vient sceller le pacte fondamental existant entre la nation et le catholicisme. 
Archambault déplore d'abord qu'il «reste des concurrents au drapeau canadien-français"3). Certains, se désole-t-il, «lui préfèrent encore le drapeau d'une nation étrangère : français, anglais, américain $[. .].]^{33}$ ». Cela constitue, à ses yeux, une «anomalie», puisque «toute nationalité a droit à son drapeau distinctif), c'est-à-dire qu'elle a besoin d'un signe "qui rallie ses enfants, qui incarne leurs aspirations communes ${ }^{94}$ ». En fait, s'il y a anomalie, c'est que, selon Archambault, la urace canadienne-française ne peut encore [...] se flatter de constituer une véritable nation», et ce, parce que "[l]a Providence a disposé autrement de nos destinées 95 \%. En somme, la nation canadienne-française doit maintenant vivre au sein d'une nation qui lui est étrangère au plan religieux et culturel.

Toujours dans le même texte, le directeur de l'ÉSP s'insurge contre ceux qui estiment que le fait de mettre le Sacré-Coeur sur le drapeau constitue un facteur de division. Au contraire, réplique-t-il, le Christ lui-même veut apparaitre sur le drapeau canadien-français parce que (il cite à cet égard Marguerite-Marie), Jésus rechercherait le «plaisir de voir les grands de la terre abaissés et humiliés devant lui, comme il a senti l'amertume de se voir anéanti à leurs pieds 99 . En d'autres termes, finie l'idée du Christ tendant la joue gauche, l'heure du pardon doit dorénavant faire place au moment du triomphe. Revanchard, le Christ doit désormais apparaitre sur le drapeau canadien-français pour montrer sa toute-puissance, car le "Christ est roi des nations comme des individus ${ }^{97}$ ), proclame Archambault. Si le Christ est roi des nations, c'est donc qu'il n'est pas seulement venu sauver les hommes mais aussi, comme le directeur de l'ÉSP l'écrit, en 1930, dans une brochure de l'ÉSP, «sauver les nations ${ }^{88}$ ". Pas de différence donc entre les âmes individuelles et les âmes nationales, toutes deux étant objet d'attention de Dieu et donc candidates à être sauvées.

Archambault tient beaucoup à cette idée puisque le 20 juin 1940, dans un article de L'Ordre nouveau, il essaie de la justifier à partir de l'exemple français. Il rappelle qu'en novembre 1918, on retrouvait assez de drapeaux du Sacré-Cœur sur le front franco-allemand, long de mille kilomètres, pour en planter un à tous les cinquante centimètres : «Qu'étaient ces drapeaux du Sacré Cœur? Le drapeau de la France sur lequel des catholiques, pour répondre à la demande de Notre-Seigneur à sainte Marguerite-Marie, avaient placé l'image de son divin Cœur ${ }^{99}$ \%). Voilà ce qui avait contribué à la victoire en 1918. Or, Archambault se réjouit, qu'au début du mois de juin 1940, le Premier ministre français d'alors, Paul Reynaud ${ }^{100}$, ait participé à une célébration religieuse à Montmartre, dans la Basili- 
que du Sacré-Cœur, présidée par le cardinal Suhart. Raison supplémentaire, aux yeux du directeur de l'ÉSP, pour qu'à la prochaine fête nationale des Canadiens français, le Sacré-Cœur se retrouve sur le drapeau canadien-français. "Il y a longtemps, écrit-il, que de fiers chrétiens en ont eu le dessein et l'ont mis à exécution. Mais le geste doit se généraliser actuellement. Il faut qu'il constitue un hommage de notre nationalité au Sacré Cœur, comme un appel collectif à sa protection». En somme, Archambault tient mordicus à ce que le lien entre le catholicisme et la nation soit signifié symboliquement sur le drapeau de la nation. En ce sens, on comprend volontiers le père Lévesque, comme il le confie dans ses Souvenances, de trouver que le directeur de l'ÉSP était un peu trop nationaliste. Car, pour Archambault, catholicisme et nationalisme s'imbriquaient l'un dans l'autre d'une manière qui ressemble à celle de Groulx. Il s'agit là du premier élément à retenir du portrait intellectuel d'Archambault.

Le second élément à retenir est qu'on retrouve également chez Archambault les principaux éléments du catholicisme social mis en lumière plus haut. C'est-à-dire un catholicisme qui, sur le fond, est bien celui des intransigeants par la lutte menée contre le libéralisme, et qui, face à «l'ennemi» de gauche se doit d'adopter une stratégie de rapprochement avec les classes populaires. Mais ce rapprochement est également accompagné d'un grand désir de changer la société. Cette refonte peut seulement être réalisée par une profonde rechristianisation de la société (un projet rappelant celui des catholiques les plus intransigeants) laquelle conduira à des changements structurels et institutionnels dont le corporatisme est le couronnement (encore que cette notion ne soit pas très bien développée par Archambault). En somme, la pensée d'Archambault se présente comme un mélange de catholicisme social et de nationalisme, le tout étant profondément ancré dans l'intransigeantisme catholique. Sa pensée oscille donc sans cesse entre catholicisme social et intransigeant, ce qui montre «'originalité» de la pensée catholique canadienne-française.

\section{NOTES}

${ }^{1} \mathrm{Ce}$ texte est une version remaniée d'une conférence prononcée au Congrès de l'Association internationale des sociologues de langue française (3-7 juillet 2000), dans le cadre du colloque intitulé «'analyse socio-historique du militantisme chrétien et personnaliste au Québec : contributions à la modernisation ambiguë de l'Église ou du Canada français, 1930-2000\%. Je tiens à remercier Sylvie Lacombe, professeuradjoint au département de sociologie de l'Université Laval, pour l'aide financière ayant rendu possible cet article.

${ }^{2}$ Georges-Henri Lévesque, Souvenances, tome I, Montréal, Éditions la Presse, 1983, p. 276. 
3Je me suis limité à la période des années trente, plus précisément aux années 19291940, parce que cette période de grande turbulence (crise économique, montée de l'extrême droite en Europe et guerre espagnole) a amené l'Église à s'impliquer grandement dans la société. Voir Martin Conway, Catholic Politics in Europe, 1918-1945, Routledge, London and New York, 1997, et plus particulièrement le chapitre trois. 'Jean-Marie Mayeur, Catholicisme social et democratie chrétienne, Paris, Cerf, 1986, p. 65. ${ }^{5}$ Lucia Ferretti, Brève bistoire de l'Église catbolique au Québec, Montréal, Boréal, 1999, p. 126.

'Émile Poulat, Église contre la bourgeoisie, Paris, Casterman, 1977.

${ }^{7}$ En 1864, le pape Pie IX fait paraitre cette encyclique où il condamne une série de propositions comme celle voulant que le Pontife romain doive se réconcilier avec le libéralisme et le progrès. En d'autres termes, le Syllabus sifflait la fin de la récréation à tous les catholiques proches du courant libéral.

'Émile Poulat, op. cit., p. 118.

'Jean-Marie Mayeur, op. cit., p. 28.

${ }^{10}$ Émile Poulat, op. cit., p. 120.

"Jean-Marie Mayeur, op. cit., p. 29

${ }^{12}$ Ibid., p. 31.

${ }^{13}$ Émile Poulat, op. cit., p. 77.

${ }^{14}$ Hugues Portelli, Les socialismes dans le discours catbolique, Paris, Le Centurion, Paris, 1986, p. 20.

${ }^{15}$ Alain Besançon, La confusion des langues. La crise idéologique de l'Église, Paris, CalmannLévy, 1978, p. 76.

${ }^{16} J$ Jean-Marie Mayeur, op. cit., p.41.

"Émile Poulat, op. cit., p. 182.

${ }^{18}$ Lucia Ferretti, op. cit., p. 135.

${ }^{19} \mathrm{Joseph}$-Papin Archambault, La menace communiste au Canada, brochures nos 254-255, Montréal, École sociale populaire, 1935, p. 3.

${ }^{20} \mathrm{Ibid}$, p. 3.

${ }^{21} \mathrm{~J}$ oseph-Papin Archambault, «Nature et but de ces Journées», Joumées anticommunistes, brochures $n^{\text {oa }}$ 251-252, Montréal, École sociale populaire, 1934-35. C'est au milieu des années trente que l'École sociale populaire lance ses Journées anticommunistes. Pour que la dénonciation de «'erreur communiste» ait un plus grand impact, on tient ces journées dans les villes les plus importantes de la province. Jean-Claude St-Amant, «La propagande de l'École sociale populaire en faveur du syndicalisme catholique, 1911-1949m, Revue dHistoire de l'Amérique française, vol. 32, $\mathrm{n}^{\circ} 2$ (septembre 1978), pp. 208-209.

${ }^{22}$ Ibid., pp. 6-7.

${ }^{23}$ Joseph-Papin Archambault, Sous la menace rouge. L'accroissement de la population cosmopolite de Montréal. Les dangers qu'il présente, les devoirs qu'il nous impose, CEuvre des tracts, $n^{\circ} 201$, mars 1936.

${ }^{24}$ Joseph-Papin Archambault, La menace communiste au Canada, op. cit., p. 13.

${ }^{25} \grave{A}$ l'occasion d'un colloque tenu à Montréal, Los intellectuels et les années trente : un brassage idéologique, ACFAS 2000, j’ai présenté une communication laquelle examinait plus en détail ce qu'Archambault, ainsi que Groulx, attendaient de l'État. ${ }^{20}$ Joseph-Papin Archambault, «Nature et but de ces Journées», loc.cit., p. 8. ${ }^{27}$ Joseph-Papin Archambault, La menace communiste au Canada, op. cit., p. 18. ${ }^{28}$ Joseph-Papin Archambault, Conférence prononcée le 2 décembre 1936 à l'Univer- 
sité d'Ottawa, dont certains extraits sont reproduits dans le journal L'Ondre nouveau, uLe communisme au Canada. Attitudes diverses», du 20 décembre 1936, p. 3. ${ }^{29}$ Ibid., p. 19.

${ }^{30}$ On consultera sur ce point Michael D. Behiels, Le Québec et la question de l'immigration : de l'ethnocentrisme an pluralisme ethnique, 1900-1985, Ottawa, Société historique du Canada, 1991, p. 7.

${ }^{31}$ Fernande Roy, Progris, Harmonie, Liberté. Le liberalisme des milieux d'affaires francophones de Montréal au tournant du siècle, Montréal, Boréal, 1988, p. 57.

${ }^{32}$ Ibid., p. 282.

${ }^{33}$ Fernande Roy, Histoire des idéologies au Québec aux $X I X '$ et $X X$ ' siècles, Montréal, Boréal, 1993, p. 92.

${ }^{34}$ Zeev Sternhell, Mario Sznajder et Maia Ashéri, «Introduction», Naissance de lidéologie fasciste, Paris, Gallimard, 1994, pp. 23-24.

${ }^{35}$ Joseph-Papin Archambault, «Déclaration d'ouverture», L'organisation professionnelle, Semaines sociales du Canada, XIV e session, Semaines sociales du Canada, Montréal, 1936, p. 21.

${ }^{36}$ Ibid, p. 21.

${ }^{37} I$ Ibid., pp. 21-22.

${ }^{38}$ Alain Besançon, op. cit., pp. 73-74.

${ }^{39} \mathrm{Joseph}$-Papin Archambault, La restauration de l'ordre social d'apris les engycliques Rerum novarum et Quadragesimo anno,, Montréal, Éditions de l'École sociale populaire, 1932, p. 82. Il s'agit là de la reproduction d'une conférence prononcée le 21octobre 1931 à l'Académie canadienne Saint-Thomas-d'Aquin à l'Université Laval à Québec

${ }^{40}$ Ibid., p. 19.

${ }^{41} I$ Ibich, p. 18.

42Joseph-Papin Archambault, «Nature et but de ces Journées», loc. cit., p. 9.

${ }^{43}$ Joseph-Papin Archambault, «Déclaration d'ouverture à la Semaine sociale», L'éducation sociale, Semaines sociales du Canada, XIII' session, Secrétariat des Semaines sociales du Canada, Montréal, 1935, p. 22.

${ }^{44}$ Ibid., p. 22.

${ }^{45}$ Joseph-Papin Archambault, «Déclaration d'ouverture», L'onganisation professionnelle, loc.cit., p. 22.

"0.Joseph-Papin Archambault, "Déclaration d'ouverture à la Semaine sociale», L'éducation sociale, loc. cit., p. 22.

${ }^{47}$ Joseph-Papin Archambault, «Des mesures de protection, oui; mais surtout des réformes sociales", [LOrdre nouveau], 5 novembre 1936, p. 1.

${ }^{48}$ Joseph-Papin Archambault, "Déclaration d'ouverture), La coopération, Semaines sociales du Canada, XV $\mathrm{XV}^{\mathrm{e}}$ session, Secrétariat des Semaines sociales du Canada, Montréal, 1937, p. 25.

49Joseph-Papin Archambault, «Déclaration d'ouverture», L'ordre social cbrítien, Semaines sociales du Canada, $\mathrm{XI}^{e}$ session, Secrétariat des Semaines sociales du Canada, Montréal,1932, p. 21.

${ }^{50}$ Joseph-Papin Archambault, «Déclaration d'ouverture», Pour une société cbrétienne, Semaines sociales du Canada, $\mathrm{XVI}^{\mathrm{e}}$ session, Secrétariat des Semaines sociales du Canada, Montréal, 1938, p. 38.

${ }^{51} J o s e p h-P a p i n$ Archambault, La restauration de l'ordre social d'apris les engycliques Rerum novarum et Quadragesimo anno, p. 95.

${ }^{52}$ Ibid, p. 52.

${ }^{53}$ Ibid., p. 53. 
${ }^{54}$ Ibid., p. 57.

55Joseph-Papin Archambault, «Nature et but de ces Journées», loc. cit., p. 10.

${ }^{56} \mathrm{~J}$ oseph-Papin Archambault, La restauration de l'ordre social d'aprìs les engycliques Rerum novarum et Quadragesimo anno, op. cit., p. 95.

${ }^{57}$ Ibid, p. 95.

58 Joseph-Papin Archambault, «Déclaration d'ouverture à la Semaine sociale», L'éducation sociale, loc. cit. pp. 27-28.

${ }^{59}$ Joseph-Papin Archambault, «Déclaration d'ouverture», L'ordre social chrétien, loc. cit., p. 23.

${ }^{60}$ Fernand Dumont, «Les années trente : la première Révolution tranquille», dans Idéologies an Canada franfais, 1930-1939, sous la direction de Fernand Dumont, Jean Hamelin et Jean-Paul Montminy, Presses de l'Université Laval, 1978, p. 9.

${ }^{61}$ On consultera à ce sujet le septième chapitre de ma thèse de doctorat, Naissance et croissance d'une unouvelle races : les présupposés philosophiques, théologiques, politiques et sociaux du nationalisme de Lionel Groulx, Ph. D. (Sciences politiques), Université Laval, 2000.

[2Joseph-Papin Archambault, «Déclaration d'ouverture à la Semaine sociale», L'éducation sociale, loc. cit., p. 19.

${ }^{63}$ Ibid., p. 21.

"64 Joseph-Papin Archambault, «Pour réaliser l'ordre nouveau. La part des instituteurs», LOrdre nouveau, 20 décembre 1936, p. 1.

${ }^{65}$ Joseph-Papin Archambault, «Déclaration d'ouverture à la Semaine sociale», L'éducation sociale, loc. cit., p. 23.

${ }^{60}$ Ibid., p. 26.

${ }^{67}$ Ibid., p. 27.

${ }^{68}$ Ibid, p. 27.

${ }^{69} \mathrm{Joseph}$-Papin Archambault, La menace communiste au Canada, p.25.

${ }^{70}$ Josepth-Papin Archambault, «Nature et but de ces Journées», Loc. cit., p. 8.

71Joseph-Papin Archambault, L'apostolat laïque, brochures n"s 202-203, L'École sociale populaire, 1930, p. 5.

${ }^{72}$ Ibid., p. 5.

${ }^{73}$ Ibid., p. 6. Dans le contexte canadien-français, Archambault pense que Jules-Paul Tardivel doit être donné en exemple.

${ }^{74} J$ oseph-Papin Archambault, L'Action catbolique au Canada, brochure n 287, L'École sociale populaire, décembre, 1937, p. 6.

${ }^{75}$ Joseph-Papin Archambault, L'Action catbolique selon les directives pontificales, École sociale populaire, 1938, p. 18. Il s'agit d'un ouvrage réalisé à partir des cours donnés par Archambault à l'Université Laval depuis 1932.

${ }^{76}$ Ibid., p. 19.

${ }^{77}$ Ibid., p. 33.

${ }^{78}$ Ibid., p. 42.

${ }^{79}$ Joseph-Papin Archambault, "Déclaration d'ouverture», L'organisation professionnelle, loc. cit., p. 23.

${ }^{80}$ Ibid., p. 29.

${ }^{81}$ Ibid., p. 29.

${ }^{82}$ Ibid., p. 23.

${ }^{83}$ Ibid., p. 23.

${ }^{84}$ Ibid., p. 24.

${ }^{85}$ Ibid., p. 25. 
${ }^{86}$ Ibid., p. 26.

${ }^{87}$ Ibid., p. 26.

${ }^{88}$ Joseph-Papin Archambault, «Déclaration d'ouverture», La coopération, loc. cit., p. 31. ${ }^{89}$ Ibid., p. 34.

${ }^{90}$ Lucia Ferretti, op. cit., p. 126.

${ }^{1} Y v e s$ Déloye, «Commémoration et imaginaire national en France (1896-1996). «France, fille aînée de l'Église, es-tu fidèle aux promesses de ton baptême?̀, Sociologie des nationalismes, Pierre Birnbaum (dir.), P.U.F., 1997, p. 63.

92 Joseph-Papin Archambault, «Le drapeau canadien-français», L'auvre des tracts, novembre 1928, p. 1. Article publié à l'occasion du vingt-cinquième anniversaire de l'adoption du drapeau.

${ }^{93}$ Ibid., p. 1.

${ }^{94}$ Ibid., p. 2.

${ }^{95}$ Ibid., p. 2.

${ }^{9}$ Ibid., p. 7

${ }^{97}$ Ibid., p. 6.

${ }^{98}$ Joseph-Papin Archambault, L'apostolat laïque, op. cit., p. 11.

${ }^{90}$ Joseph-Papin Archambault, "Tous pour la victoire», LOrdre nouveau, 20 juin 1940.

${ }^{100} \mathrm{Il}$ cédera sa place au maréchal Pétain peu avant le 20 juin de cette année-là. 